\title{
Differentially Expressed Genes Associated with Human Limbal Epithelial Phenotypes: New Molecules That Potentially Facilitate Selection of Stem Cell-Enriched Populations
}

\author{
Lili Takács, ${ }^{1}$ Enikó Tóth, ${ }^{1,2}$ Gergely Losonczy, ${ }^{1}$ Attila Szanto, ${ }^{3}$ Tomi Bähr-Ivacevic, ${ }^{4}$ \\ Vladimir Benes, ${ }^{4}$ András Berta, ${ }^{1}$ and György Vereb ${ }^{2}$
}

Purpose. The aim of this study was to identify differentially expressed genes in the human limbal epithelium by microarray analysis.

Methods. Total RNA isolates of human limbal and central corneal epithelia were used after transcription for hybridization on whole human genome expression microarrays. A set of differentially expressed genes detected by both microarrays was established. In the case of eight selected molecules, microarray results were confirmed by qRT-PCR, and protein expression in the cornea was examined by confocal immunofluorescence microscopy. Colocalization with the putative stem cell marker $\mathrm{C} / \mathrm{EBP} \delta$ was also examined.

REsults. The authors established a database of 126 limbal overexpressed genes. qRT-PCR confirmed microarray results in all examined cases (SPON1, IFITM1, ITM2A, PHLDA1, CXCR4, FZD7, DCT, DKK4). Limbal localization of the protein product of SPON1, IFITM1, ITM2A, CXCR4, and DKK4 was shown with confocal immunofluorescence microscopy. SPON1, IFITM1, and ITM2A signals mostly colocalized with C/EBP $\delta$-positive putative resting limbal stem cells

Conclusions. By detecting several new differentially expressed genes in the human corneal limbus, this study further expands current knowledge on the molecular signature of limbal epithelial stem cells. Plasma membrane localization of IFITM1 and ITM2A suggests their potential usefulness as targets to select stem cell-enriched populations from the limbal epithelium. (Invest Ophthalmol Vis Sci. 2011;52:1252-1260) DOI: 10.1167/iovs.10-5242

Gxperimental evidence and clinical observations indicate Chat corneal epithelial stem cells reside in the corneal
that limbus, ${ }^{1}$ in the specific epithelial structures designated as limbal palisades of Vogt. ${ }^{2,3}$ Damage to the limbal tissues may lead

From the Departments of ${ }^{1}$ Ophthalmology, ${ }^{2}$ Biophysics and Cell Biology, and ${ }^{3}$ Biochemistry and Molecular Biology, Medical and Health Science Center, University of Debrecen, Debrecen, Hungary; and ${ }^{4}$ EMBL Genecore, Heidelberg, Germany.

Supported by Hungarian National Research Fund Grants OTKA F046321, K75752, K68616, and TAMOP-4.2.1/B-09/1/KONV-2010 0007.

Submitted for publication January 20, 2010; revised May 7 and September 19, 2010; accepted October 10, 2010.

Disclosure: L. Takács, None; E. Tóth, None; G. Losonczy, None; A. Szanto, None; T. Bähr-Ivacevic, None; V. Benes, None; A. Berta None; G. Vereb, None

Corresponding author: Lili Takács, Department of Ophthalmology, Medical and Health Science Center, University of Debrecen, Nagyerdei krt. 98, Debrecen H-4012, Hungary; ltakacs@dote.hu. to limbal stem cell deficiency (LSCD), conjunctivalization of the corneal surface, and severe visual loss. In recent years, transplantation of cultured limbal epithelial stem cells resulted in a breakthrough in the therapy of LSCD. ${ }^{4,5}$ However, many aspects of the ocular surface reestablishment after corneal stem cell transplantation are unclear. A better knowledge of the molecules expressed by limbal epithelial cells could help our understanding on stem cell functions, increase our comprehension of the mechanisms involved in ocular surface regeneration, and, finally, improve the success rate of limbal stem cell transplantation. ${ }^{6}$

The spatial separation of limbal stem cells from transient amplifying and differentiated cells in the central cornea allows for the preparation of a stem cell-containing population. ${ }^{7}$ Transcriptional profiling of stem cell-enriched populations has proven to be effective in determining a stem cell signature in the epidermis. ${ }^{89}$ Recently, microarray analysis of limbal epithelial cells has led to the identification of several new molecules that are preferentially expressed in the limbal epithelium. ${ }^{10,11}$

Despite intensive research, a definitive limbal epithelial stem cell marker is still lacking. ABCG2, a xenobiotic transporter molecule, has been associated with stem cells in several tissues, including the limbal epithelium. ${ }^{12-14}$ One isoform of the p63 transcription factor, $\Delta \mathrm{Np} 63 \alpha$, is specific for activated, highly proliferative corneal epithelial stem cells. ${ }^{15,16}$ After corneal wounding and activation of limbal stem cells, $\Delta \mathrm{Np} 63 \alpha$ positive cells may appear in multiple layers of the limbal epithelium and even in the peripheral cornea. ${ }^{16}$ In a recent study, Bmi-1, a polycomb complex protein found in several adult stem cell types, was proposed as a limbal epithelial stem cell marker based on mRNA expression analysis. ${ }^{17}$ Another study showed that CCAAT enhancer binding protein C/EBP $\delta$ and Bmi-1 colocalized with a subpopulation of $\Delta \mathrm{Np} 63 \alpha$-positive limbal basal epithelial cells and identified quiescent cells that formed holoclones in culture. ${ }^{18}$ It is considered now that $\Delta \mathrm{Np} 63 \alpha$ sustains the proliferative potential, whereas $\mathrm{C} / \mathrm{EBP} \delta$ regulates the self-renewal and mitotic rate of limbal stem cells. ${ }^{18,19}$

The aim of the present study was to explore differentially expressed genes in the human limbal epithelium to characterize the limbal epithelial phenotype and reveal new molecules potentially associated with the limbal epithelial stem cells. Several previously unreported genes were found to be upregulated in limbal samples, and differential expression of selected molecules was confirmed by qRT-PCR and immunofluorescence staining. Colocalization with $\mathrm{C} / \mathrm{EBP} \delta$ was used to examine the relationship of the newly revealed molecules to putative quiescent limbal epithelial stem cells. Purposefully, several 
membrane-bound molecules were chosen for validation studies to identify potential selection targets for flow cytometry.

\section{Materials ANd Methods}

\section{Corneal Samples and Total RNA Isolation}

All examinations and sample collections were conducted according to the tenets of the Declaration of Helsinki and followed the guidelines of our Institutional Ethics Committee. Corneas of nine eyes enucleated for uveal melanoma were used for mRNA isolation. In three cases, central and limbal regions of the same cornea were used (these samples are referred to as matching pairs in the rest of this article). In six other cases, the central 7-mm corneal button was excised for corneal transplantation before mRNA isolation; in these cases, only the limbal region was used. Central corneal epithelia removed during photorefractive keratectomy (PRK) were also collected from 17 patients. From the three whole corneas of enucleated eyes, the central 7-mm corneal button was excised with a trephine. Conjunctival remnants were cut with scissors from the limbal rims. The epithelium was scraped from the cornea and the limbus was scraped with a hockey knife under an operating microscope. To avoid expression changes during tissue culture or mRNA damage because of repeated freeze-thaw cycles, all epithelial samples were scraped and placed into lysis buffer (Qiagen, Valencia, CA) complemented with $\beta$-mercaptoethanol within 2 hours after enucleation or immediately after removal during PRK. Total RNA was then isolated from the samples using silica-membrane columns (RNeasy Mini Kit; Qiagen) according to the manufacturer's instructions. RNA quality and quantity were checked (Bioanalyzer Nano Chips; Agilent Technologies, Santa Clara, CA). The six best central and limbal samples were selected and used for microarray hybridization experiments

\section{cRNA Preparation and Microarray Analysis}

One microgram of total RNA was used to prepare labeled cRNA (CodeLink Expression Assay Reagent Kit; GE Healthcare, Piscataway, NJ). Reactions from all samples yielded sufficient cRNA for subsequent microarray analysis. To minimize annotation-related errors, samples were hybridized to two different microarrays, both representing the total human genome. cRNA samples from six limbal and six central corneas (among them three matching pairs) were hybridized on 30mer microarrays (CodeLink Human Whole Genome; GE Healthcare). The three matching central and limbal cornea pairs were also hybridized on 25-mer microarrays (U133 Plus 2.0; Affymetrix, Santa Clara, CA). Fragmentation and hybridization were carried out according to the standard protocols provided for each microarray by the manufacturer. Microarrays were scanned, the size and morphology of each spot were examined, and low-quality spots were masked. Only scans with more than 99\% appropriate spots were further analyzed. Spots were gridded and assigned, and the intensity values were calculated according to the manufacturer's standard protocol. The resultant data were then normalized on a per chip, and subsequently on a per gene, basis. Normalized data were imported in a data analysis software package (GeneSpring 7; Agilent Technologies).

\section{Data Analysis}

Targets with a normalized expression level above 0.6 were considered as present for the GE Healthcare chips, whereas tagging for the Affymetrix chip was performed with image data analysis software (GeneChip Operating Software; Affymetrix) using the MAS5 algorithm. For each microarray, two datasets were generated, one representing the limbal and the other representing the central corneal samples. Genes with at least twofold changes were considered as upregulated or downregulated (differentially expressed genes [DEGs]). ANOVA at a significance level of $P=0.005$ was used to minimize false discovery. A list of targets identified as DEGs by both the GE Healthcare and Affymetrix platforms was generated. To compare the datasets of dif- ferentially expressed genes created using these platforms, it was necessary to uncover targets representing identical genes on the different microarrays. Sequence-based comparison between these two platforms was not feasible because oligonucleotide sequence information was available only for the Affymetrix microarray. Available sequence identifiers (GenBank and RefSeq) were used to search Entrez, Ensembl, and Swissprot databases, and targets with at least one matching identifier were considered identical.

DEGs identified by both microarrays were further analyzed to point out molecules that are possibly associated with the limbal stem cell phenotype. We looked for DEGs that were absent or marginal in the central cornea with at least threefold higher expression in the limbal epithelium, showing little variation between individual samples $(P<$ $0.005)$. In addition, a PubMed search was performed for each DEG, and those involved in stem cell biology or corneal development were chosen for further studies. Several membrane-bound molecules were selected to identify possible selection markers.

\section{qRT-PCR}

Nine limbal and 20 central RNA samples were reverse transcribed with random hexamers (cDNA High Capacity Archive Kit; Applied Biosystems, Foster City, CA). cDNAs were analyzed with quantitative realtime PCR using verified assays (TaqMan; Applied Biosystems) for the individual genes and master mix (TaqMan; Applied Biosystems). All samples were analyzed in triplicates on a real-time PCR machine (ABI7900; Applied Biosystems). Template-free and reverse transcriptase-free controls were used to determine nonspecific signals. Ct values for each well were determined (Sequence Detection Software; Applied Biosystems), and the expression value was calculated as $\left[2^{38.5-\mathrm{Ct}(\mathrm{gene})}\right]$. Then the mean of the replicates was calculated for each gene (including all target genes and two housekeeping genes, GAPDH and $\beta$-actin) in each tissue sample. Normalization per sample was achieved by dividing the average expression $\left[2^{38.5-\mathrm{Ct}(\mathrm{gene})}\right]$ of target genes by that of a housekeeping gene. Normalized expressions for each gene were averaged for all samples in both groups and plotted as mean \pm SEM on $\log$ scale. Statistical significance of the differences in normalized expression between the nine limbal and 20 central corneal samples was tested using Student's $t$-test with correction for unequal variances.

\section{Immunofluorescence}

Three cadaveric corneas, unsuitable for corneal transplantation, were provided by the Cornea Bank of Debrecen. These were cut into quadrants. Each quadrant was placed in a tissue-embedding system (TissueTek; Sakura Finetek, Tokyo, Japan), frozen in liquid nitrogen, and then stored at $-80^{\circ} \mathrm{C}$. Samples were oriented so that the cutting plane dissected obliquely the limbal region. Cryostat sections (8-10 $\mu \mathrm{m}$ ) were placed on electrostatically charged slides (Superfrost; Fischer Scientific, Pittsburgh, PA). Sections were fixed in chilled acetone and air dried. Before immunofluorescence staining, sections were rehydrated in PBS containing 0.5\% BSA and $0.05 \%$ nonionic surfactant (Triton-X-100; Sigma-Aldrich, St. Louis, MO). Slides were incubated with $50 \mu \mathrm{L}$ primary antibody solution for 1 hour at room temperature. The following primary antibody dilutions were used: rabbit anti-C/ EBP $\delta$ 1:400, mouse anti-C/EBP $\delta$ (clone 92.69) 1:100 (Santa Cruz Biotechnology, Santa Cruz, CA), rabbit anti- $\Delta$ Np63 1:50 (BioLegend), mouse anti-ABCG2 clone BXP21 1:15 (Affinity Bioreagents, Golden, CO), mouse anti-Bmi-1 (clone F6) 1:250 (Millipore/Upstate, Temecula, CA), goat anti-IFITM1 1:200, goat anti-ITM2A 1:100, goat anti-DKK4 1:20, goat anti-F-spondin1 1:100 (all from Santa Cruz), and rabbit anti-CXCR4 (Affinity Bioreagents) 1:200. After three washes in PBS/ $0.5 \% \mathrm{BSA} / 0.05 \%$ nonionic surfactant (Triton-X-100) slides were incubated for 1 hour at room temperature with $50 \mu \mathrm{L}$ second antibody cocktail containing $1-\mu \mathrm{g} / \mathrm{mL}$ solutions of donkey anti-goat-AlexaFluor488, (from Molecular Probes/Invitrogen, Eugene, OR), donkey anti-rabbit-Cy3, and donkey anti-mouse-Cy5 (both from Jackson ImmunoResearch, West Grove, PA). Isotype controls and controls stained with the secondary antibodies only were prepared with each staining 
TABLE 1. Top 50 Genes Overexpressed in Limbal Epithelium

\begin{tabular}{ll}
$\begin{array}{l}\text { Common } \\
\text { Name }\end{array}$ & \multicolumn{1}{c}{ Product } \\
\hline Development/Differentiation \\
ITM2A & Integral to membrane 2A \\
SEMA6A & Semaphorine 6A \\
SOX9 & Transcription factor SOX9 \\
CDH19 & Cadherin 19, type 2 preproprotein \\
VLDLR & Very low density lipoprotein receptor isoform a \\
TCF4 & Transcription factor 4 \\
ETS2 & v-ets erythroblastosis virus E26 oncogene homolog 2 \\
DKK 4 & Dickkopf homolog 4 precursor
\end{tabular}

\section{Extracellular Matrix Components}

$\begin{array}{ll}\text { TFPI2 } & \text { Tissue factor pathway inhibitor } 2 \\ \text { SPON1 } & \text { Spondin-1 } \\ \text { SPARCL1 } & \text { SPARC-like 1 } \\ \text { COL3A1 } & \text { Collagen, type III, alpha 1 preproprotein }\end{array}$

IFITM2

VEGFC Interferon induced transmembrane protein 2 Interferon induced transmembrane protein 1 (9-27)

Vascular endothelial growth factor C preproprotein

\section{Immune/Inflammatory Response}

CXCL2 Chemokine (C-X-C motif) ligand 2

S100A8 S100 calcium-binding protein A8

IL8 Interleukin 8 precursor

CXCR4 Chemokine (C-X-C motif) receptor 4 isoform b

FCER1A FC fragment of IgE, high affinity I, receptor for; alpha polypeptide precursor

\section{Melanin Biosynthesis}

$\begin{array}{ll}\text { TYR } & \text { Tyrosinase precursor } \\ \text { DCT } & \text { Dopachrome tautomerase } \\ \text { TYRP1 } & \text { Tyrosinase-related protein 1 } \\ \text { SILV } & \text { Silver homolog }\end{array}$

$e^{-}$Transport, Metabolism

$\begin{array}{ll}\text { FMO2 } & \text { Flavin containing mono-oxygenase } 2 \\ \text { DPYSL3 } & \text { Dihydropyrimidinase-like } 3 \\ \text { FMO1 } & \text { Flavin containing mono-oxygenase } 1 \\ \text { NOS2A } & \text { Nitric oxide synthase 2A isoform } 1 \\ \text { CYP24A1 } & \text { Cytochrome P450, family 24 precursor } \\ \text { CA12 } & \text { Carbonic anhydrase XII isoform 1 precursor } \\ \text { CH25H } & \text { Cholesterol 25-hydroxylase }\end{array}$

$\mathrm{CH} 25 \mathrm{H}$

Cholesterol 25-hydroxylase

NM_00208

NM_002964

NM_000584

NM_003467

NM_002001

NM_000372

NM_000550

NM_006928

NM 007177

NM_001387

NM_002021

NM_000625

NM 000782

NM_017689

NM_003956

NM_148957

Tumor necrosis factor receptor superfamily, member 19
Pleckstrin homology like domain, family A, member 1 BC039558

PHLDA1

\section{Signal Transduction}

$\begin{array}{ll}\text { RGS1 } & \text { Regulator of G-protein signaling } 1 \\ \text { GRM3 } & \text { Glutamate receptor, metabotropic } 3 \text { precursor } \\ \text { TRPM1 } & \text { Transient receptor potential cation channel, subfamily M, member } 1\end{array}$

NM_002922

NM 000840

NM_002420

NM_002275

NM_002274

NM_002276

NM_003380

BX103355

NM_145171

BT007074

GPHB5

GPNMB

$M A M D C 2$
Glycoprotein alpha 2

Glycoprotein (transmembrane) $\mathrm{nmb}$

MAM domain containing 2
NM_006528

NM_006108

NM_004684

NM 000090

NM 007177

NM_032918

H25356

NM_003641

NM_005429

NM_001922

$\begin{array}{rl}13.37 & 0.0000214 \\ 9.86 & 0.00000023 \\ 7.65 & 0.000244 \\ 7.03 & 0.000403 \\ 5.95 & 0.00284 \\ 5.00 & 0.00397 \\ 4.95 & 0.00468 \\ 3.06 & 0.00408\end{array}$

$\begin{array}{ll}20.9 & 0.0003 \\ 17.12 & 0.00000000611 \\ 10.13 & 0.000311 \\ 8.69 & 0.0000366\end{array}$

$8.69 \quad 0.0000366$

$\begin{array}{rl}14.31 & 0.000342 \\ 10.00 & 0.00000466 \\ 8.83 & 0.0000206 \\ 8.67 & 0.000113 \\ 5.80 & 0.000034 \\ & \\ & \\ 49.9 & 0.000111 \\ 46.25 & 0.000168 \\ 15.34 & 0.000236 \\ 7.14 & 0.000356 \\ 5.74 & 0.0011\end{array}$

0.0000399 0.00000829 0.00122 0.0000218

0.000291

14.31

0.00131

0.00466

0.000118

0.0000109

7.24

0.000927

$\begin{array}{ll}12.57 & 0.000318 \\ 12.20 & 0.0000557\end{array}$

$\begin{array}{rl}10.51 & 0.00137 \\ 5.89 & 0.000351 \\ 5.15 & 0.000153\end{array}$


Table 1. (continued). Top 50 Genes Overexpressed in Limbal Epithelium

\begin{tabular}{|c|c|c|c|c|}
\hline $\begin{array}{c}\text { Common } \\
\text { Name }\end{array}$ & Product & GenBank ID & Fold Change & $\boldsymbol{P}$ \\
\hline RHOBTB1 & Rho-related BTB domain containing 1 & NM_014836 & 7.04 & 0.000208 \\
\hline$P L X N C 1$ & Plexin C1 & AI290473 & 6.34 & 0.000683 \\
\hline TMEM47 & Transmembrane 4 superfamily member 10 & NM_031442 & 6.17 & 0.0000773 \\
\hline Unknown & Unknown & AI825409 & 5.63 & 0.0000726 \\
\hline FLJ00204 & FLJ00204 protein & AK074131 & 5.05 & 0.000628 \\
\hline
\end{tabular}

experiment. One rehydrated sample of each cadaveric cornea treated only with the washing buffers was examined for tissue autofluorescence. Cell nuclei were counterstained with DAPI. After three times washes, sections were fixed in $1 \%$ paraformaldehyde in PBS, then washed and mounted in mounting medium (Mowiol; Polysciences, Warrington, PA). Sections were examined with a laser scanning confocal microscope (LSM510; Zeiss, Thornwood, NY) in multitrack mode to prevent channel cross-talk. The same settings were used throughout for each labeled protein; gain was adjusted to the fluorescence signal from each protein, whereas offset was always 0 . Negative controls shown were isotype controls and imaged with the highest gain setting (used for the protein giving rise to the lowest fluorescence signal) in each channel.

\section{Results}

When six limbal mRNA samples were compared with six central cornea samples on the GE microarray (CodeLink; GE Healthcare), 494 DEGs were found. Analysis of three matching limbal and central mRNA samples on the Affymetrix microarray (U133 plus 2.0; Affymetrix) yielded 745 DEGs. The correlation coefficient between these two datasets was 0.715 . One hundred twenty-eight transcripts, representing 126 genes, showed statistically significant, at least twofold overexpression in limbal samples according to both microarrays. The top 50 of these molecules, grouped according to molecular function, are listed in Table 1. The mRNA for dopachrome tautomerase, tyrosinase, and tyrosinase-related protein 1 that are related to melanogenesis, as well as mRNAs for molecules related to the Wnt pathway (frizzled 7 , dickkopf homolog 4 , transcription factor 4 , very low-density lipoprotein receptor), were found to be prominently higher in the limbus. The mRNA levels of two currently used putative limbal stem cell markers, ABCG2 and $\Delta \mathrm{Np} 63 \alpha$, were also elevated in limbal samples; however, both differed by a factor less than 2 . The difference in mRNA levels of two recently suggested limbal stem cell-associated molecules, Bmi- 1 and $\mathrm{C} / \mathrm{EBP} \delta$, did not fulfill our criteria of differential expression either (data not shown). Eight genes have been selected for further examination and confirmation of microarray results. The differential expression of all examined molecules (interferon-induced transmembrane protein 1 [IFITM1], integral to membrane 2A [ITM2A], dopachrome tautomerase [DCT], pleckstrin homology-like domain, family A, member 1 [PHLDA1], chemokine C-X-C motif receptor 4 [CXCR4], spondin 1 [SPON1], dickkopf homolog 4 [DKK4], frizzled 7 [FZD7]) has been confirmed by qRT-PCR (Fig 1 ). The respective $P$ values were $0.0002,0.004,0.027,0.014,0.002,0.024,0.076$, and 0.004 for values normalized to GAPDH. $\beta$-Actin message, as control, was statistically identical in the central and limbal sets when normalized to GAPDH $(P=0.98)$. Equivalent results were obtained when $\beta$-actin was used for normalization.

The localization of five molecules has been examined by immunofluorescence in cadaveric cornea samples (Fig. 2). IFITM1 localized to clusters of cells in the basal layer of the limbal epithelium and some limbal stromal cells. ITM2A and SPON1 stained clusters of basal limbal epithelial cells and single suprabasal cells. Antibodies to CXCR4 and DKK 4 stained the membrane of basal and suprabasal limbal epithelial cells. Localization of these molecules in the central cornea was also examined. CXCR4 could be detected in basal central corneal cells; the other molecules were faintly visible in the peripheral cornea and disappeared in the central cornea. DKK4 was not observable in either the central or the peripheral cornea. The staining of ITM2A in central corneas has been shifted from the cytoplasmic membrane to the nucleus.

To elucidate the relationship between the above molecules and putative limbal epithelial stem cells, colocalization with the stem cell-associated molecule $\mathrm{C} / \mathrm{EBP} \delta$ was used. To demonstrate its relevance, $\mathrm{C} / \mathrm{EBP} \delta$ immunofluorescence was compared with the other accepted limbal stem cell markers ABCG2 and $\Delta \mathrm{Np} 63$ and the suggested marker Bmi-1 (Fig. 3). C/EBP $\delta$ staining was present in the nuclei of basal limbal epithelial cell clusters and could not be observed in the central cornea. Bmi-1 almost totally colocalized with $\mathrm{C} / \mathrm{EBP} \delta$ in the basal limbal epithelium; however, nuclei of the superficial epithelial cells were also positive for Bmi-1. C/EBP $\delta$ staining colocalized with basal limbal cells, showing membranous ABCG2 staining. In addition, C/EBP $\delta$ identified a subpopulation of $\Delta \mathrm{Np} 63$-positive cells (Fig. 3). $\Delta$ Np63 also colocalized with ABCG2, but it also showed positivity in suprabasal cells with cytoplasmic ABCG2 staining.

Labeling with IFITM1 colocalized with C/EBP $\delta$ signals in most of the cells. ITM2A- and SPON1-positive cells showed $\mathrm{C} / \mathrm{EBP} \delta$ staining in $50 \%$ to $70 \%$, indicating that these molecules are expressed in putative quiescent limbal epithelial stem cells and in their close proximity. DKK4- and CXCR4-positive cells

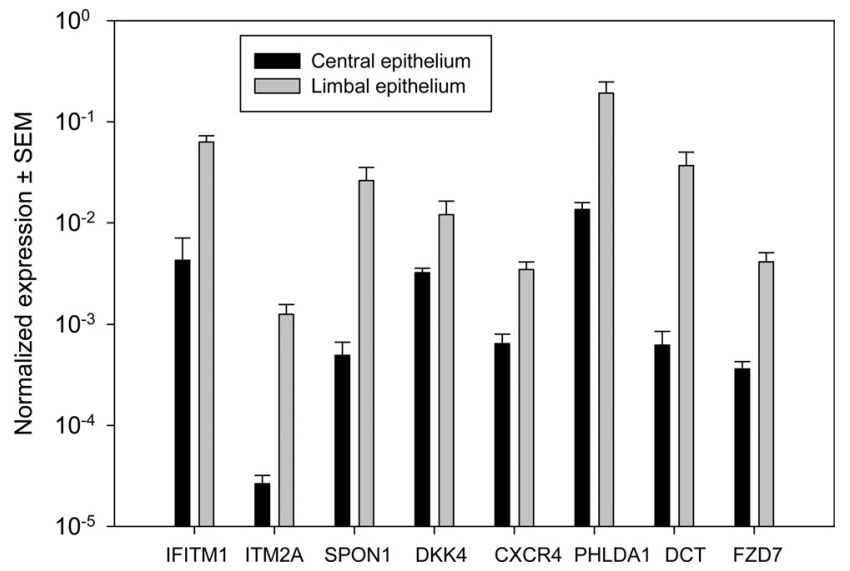

FIGURE 1. qRT-PCR validation of selected differentially expressed genes. To validate microarray results, mRNA levels of SPON1, IFITM1, ITM2A, DKK4, CXCR4, PHLDA1, FZD7, and DCT were measured by qRT-PCR in total RNA isolates of limbal (black) and central (gray) corneal epithelial samples. Data were calculated relative to GAPDH expression levels and are shown on a logarithmic scale. Columns represent the mean \pm SEM values for 9 limbal and 20 central epithelial samples. qRT-PCR measurements substantiated limbal overexpression of all examined molecules, in accordance with microarray results. 
central cornea

IFITM1
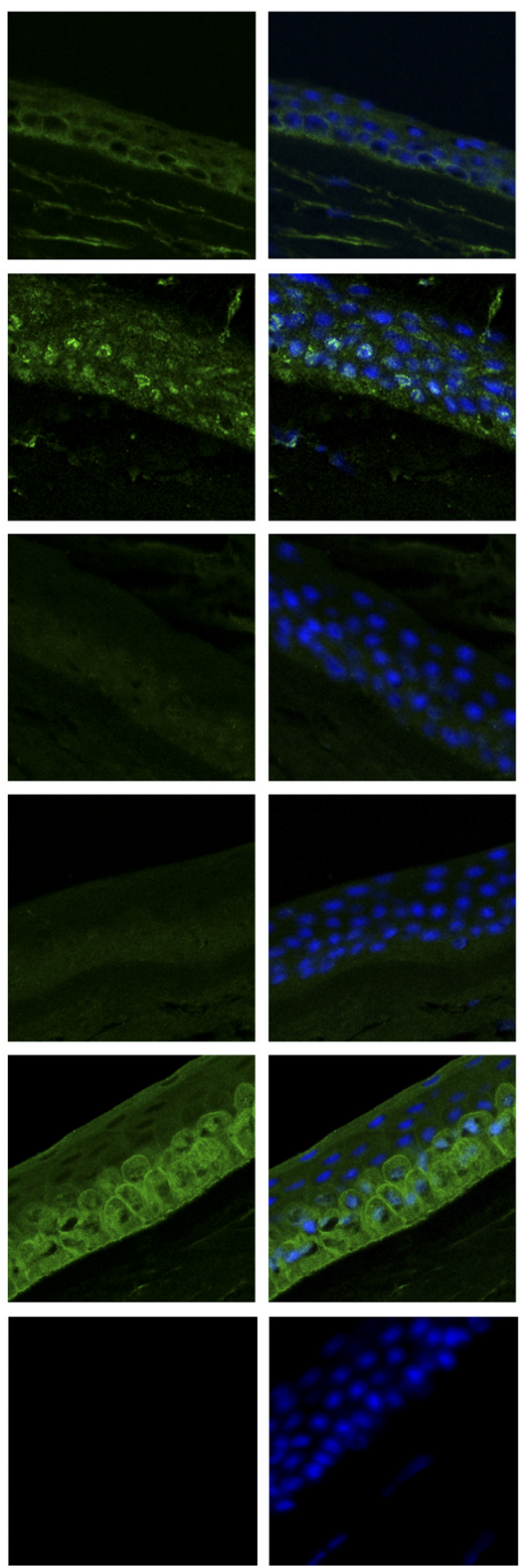

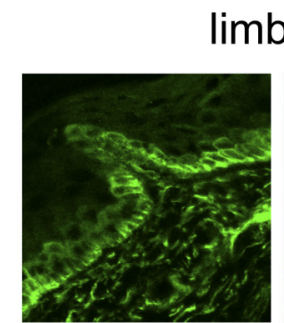

limbus
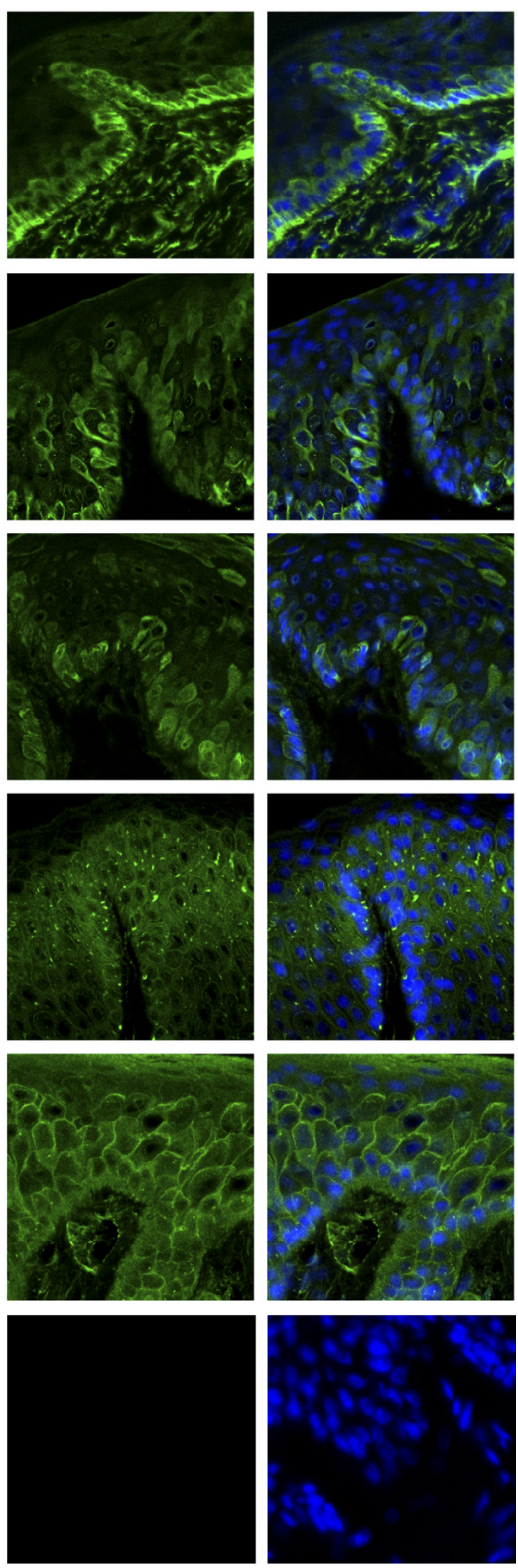

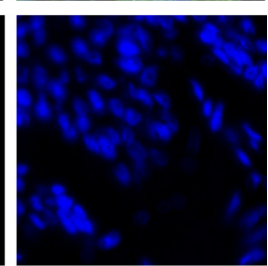

Figure 2. Confocal immunofluorescence microscopy of IFITM1, ITM2A, SPON1, DKK4, and CXCR4 in central and limbal corneal samples. Blue: nuclear staining with DAPI. Green: immunofluorescent protein label. Control, isotype control with nuclear stain only. IFITM1, ITM2A, and SPON1 label is seen in clusters of limbal basal epithelial cells; some sporadic suprabasal cells are also stained with ITM2A and SPON1 antibodies. No membrane staining with IFITM1 and ITM2A is seen in the central cornea, although very faint cytoplasmic IFITM1 and nuclear ITM2A staining can be observed. SPON1 was not detected in the central corneal epithelium. DKK 4 label is seen in basal and suprabasal limbal epithelial cell membranes and is absent in central corneal epithelium. CXCR4 label is present in basal and suprabasal limbal epithelial cells and in basal cells of the central corneal epithelium. Images are from a $110 \times 110-\mu \mathrm{m}$ field of view. included those with $\mathrm{C} / \mathrm{EBP} \delta$ staining but were present in more limbal epithelial cells, indicating that they stained early TACs and putative stem cells (Fig. 4).

\section{Discussion}

The limbal epithelial compartment contains many different cell types, including stem cells, transient amplifying and differentiated epithelial cells, and immune-related cells and melanocytes. Our goal was to identify, using mRNA microarray analysis, possible stem cell-associated molecules coded by genes overexpressed in the limbus. As occurred in other studies, ${ }^{20}$ among the most prominently overexpressed genes we found were several (dopachrome tautomerase, tyrosinase, tyrosinaserelated protein 1) related to melanogenesis. Several differentially expressed molecules were related to the Wnt pathway (frizzled 7, dickkopf homolog 4, transcription factor 4 , very low-density lipoprotein receptor). This confirms the significance of Wnt signaling in corneal epithelial differentiation and indicates that relatively rare limbal cells, such as melanocytes, may yield enough specific mRNA to be revealed in the limbal mRNA profile.

Stem cells represent a very small fraction of limbal epithelial cells, probably only between $0.5 \%$ and $1 \%$. Therefore, the contribution of stem cell-related transcripts to the limbal epithelial RNA profile is relatively small. This may be the reason many known stem cell-associated molecules, such as ABCG2, $\mathrm{p} 63, \mathrm{C} / \mathrm{EBP} \delta$, and Bmi-1, were not identified as DEGs, exceeding a $2 \times$ factor in our study and in several other reported limbal gene expression analyses. ${ }^{10,11,20}$ To reveal these molecules as DEGs, the examined cell population should probably contain stem cells in higher proportions than unsorted cells from limbal epithelial scrapings. Accordingly, mRNA levels of $\mathrm{ABCG} 2, \mathrm{C} / \mathrm{EBP} \delta,{ }^{21}$ and $\mathrm{Bmi}-1^{17}$ were found to be significantly increased on flow cytometric selection of the side population cells that are believed to carry stem-like properties. Selection of the side population, however, is a time-consuming procedure, during which expression profiles can change significantly. In 

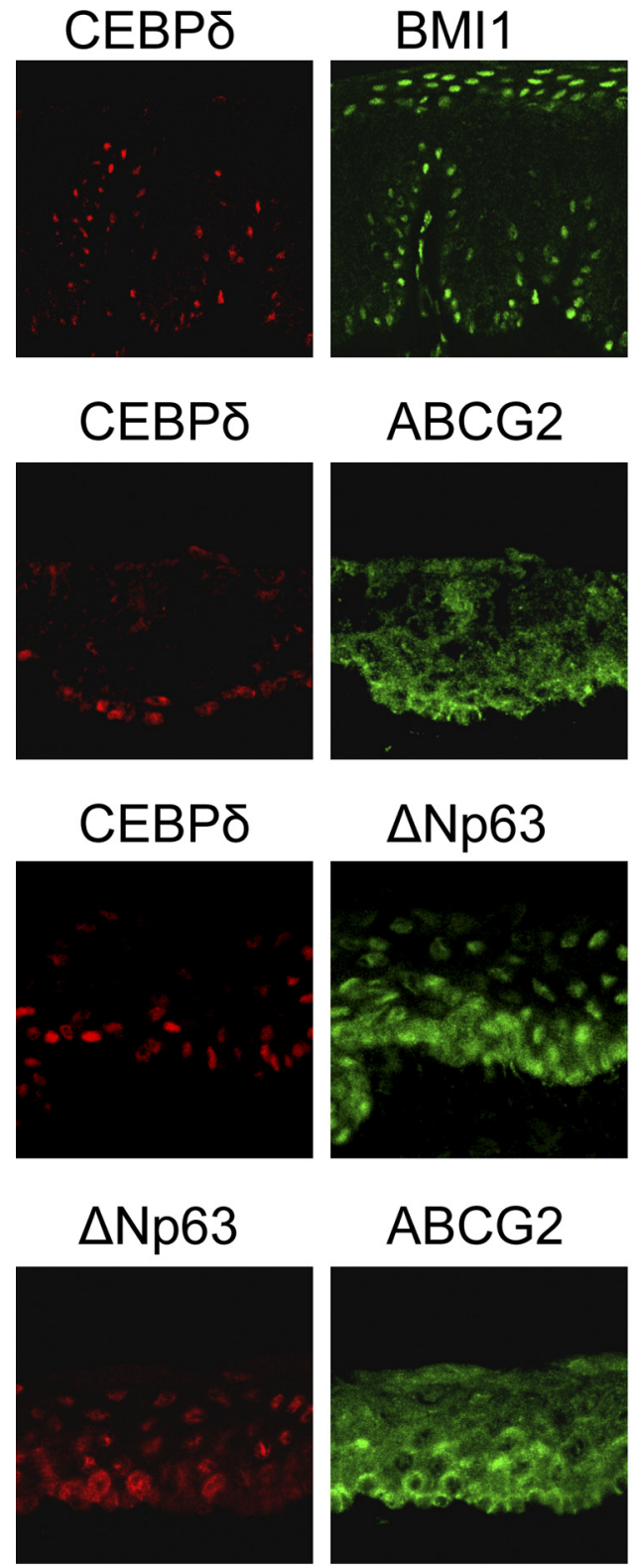
tently present in the nuclei of basal limbal epithelial cells and there colocalizes with membranous ABCG2 staining (second row). In addition, C/EBP $\delta$ identifies a subpopulation of $\Delta$ Np63-positive cells (third row). $\Delta \mathrm{Np} 63$ also colocalizes with ABCG2 but shows positivity in suprabasal cells with cytoplasmic ABCG2 staining as well (fourth row). Isotype controls with DAPI staining are imaged with the highest gain settings in each column (last row). Images are from $110 \times 110-\mu \mathrm{m}$ fields of view, except first and last rows $(160 \times 160 \mu \mathrm{m})$.
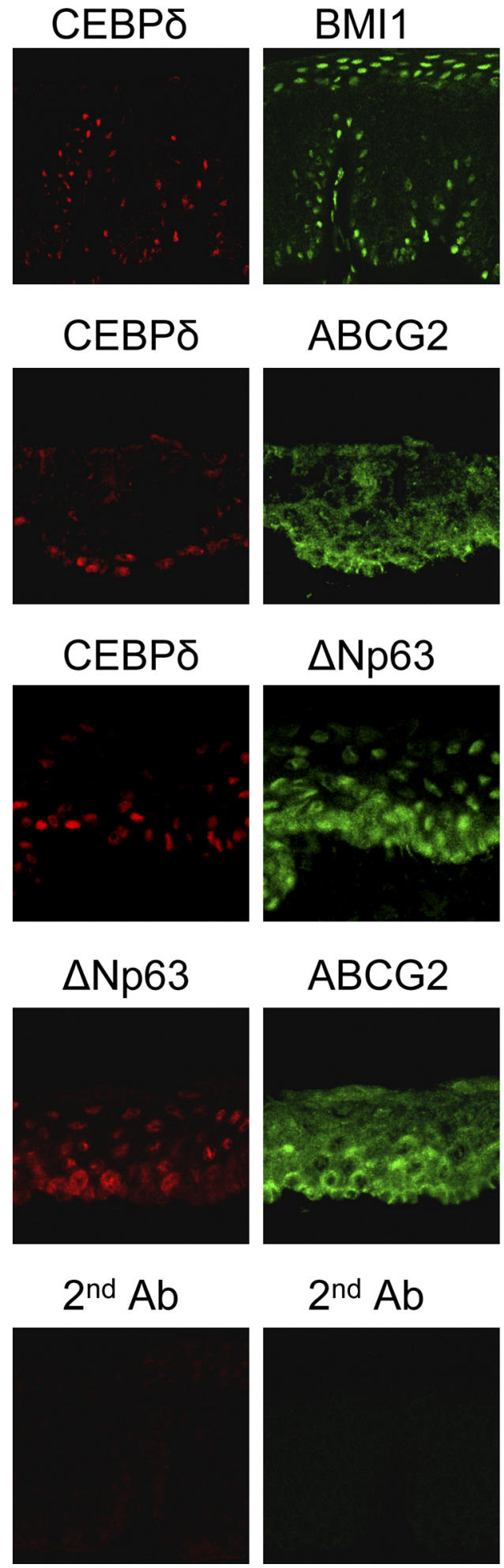
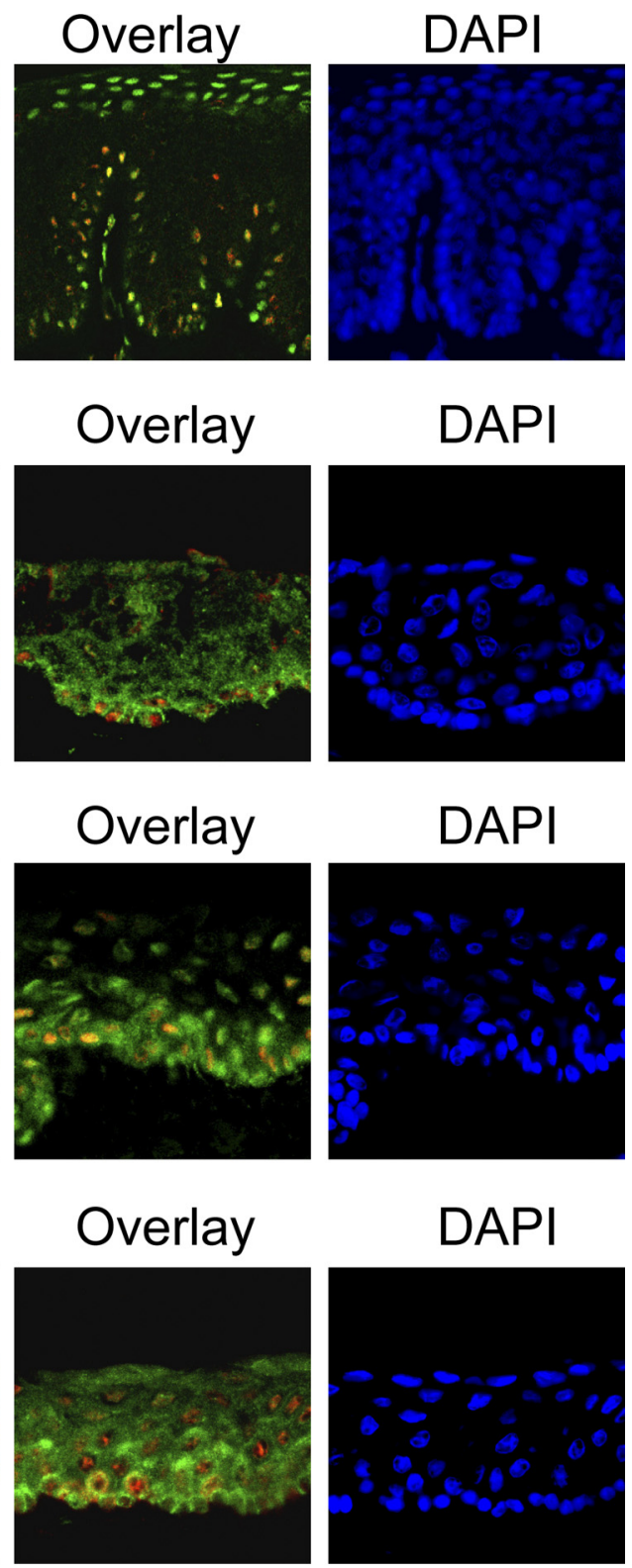

addition, the limited availability of human limbal tissue for research purposes calls for other approaches. We attempted to identify possible stem cell-associated molecules among the limbal overexpressed genes by stringent data analysis and thorough literature mining. We have narrowed the list of genes according to the following criteria: (1) the expression is significantly different between the central epithelium and the limbus, and the differences are reliably and repeatedly seen in many samples; (2) the expression differs greatly, minimally by a factor of 3, producing high levels of mRNA in the limbus and low or no levels in the central cornea; (3) the gene product should be related to stem cell homeostasis/signaling (maintenance of stemness, accepting signals from the stem cell niche, early differentiation of stem cell-derived offsprings), or there should be previous evidence that it has a role in corneal differentiation or regeneration; (4) cell membrane-associated molecules should be among them, because, if proven to be dominantly present in stem cells, they could serve as selection 


\section{CEBPס}
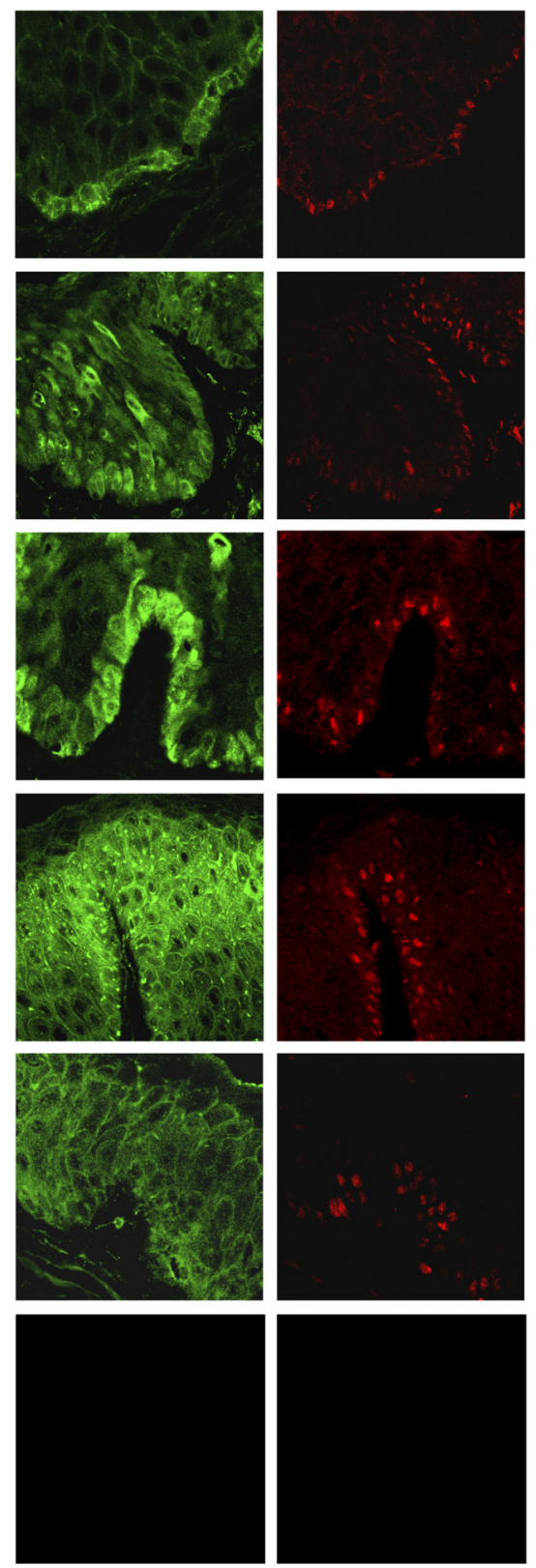

Overlay
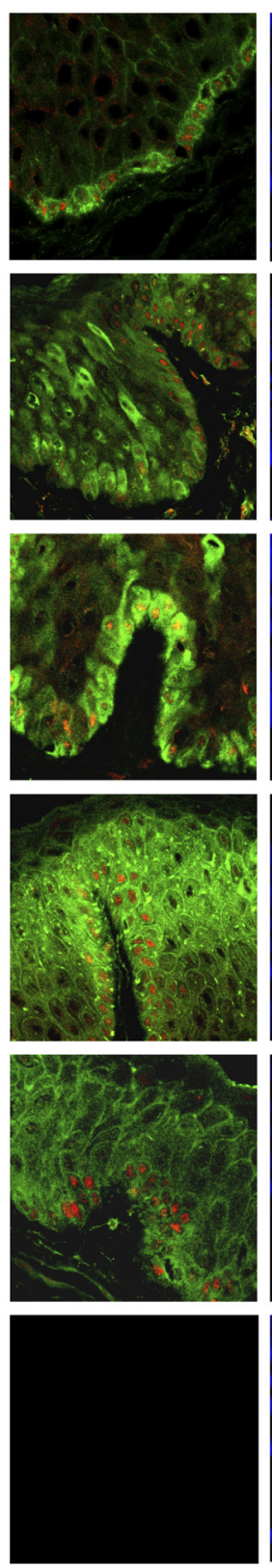

DAPI
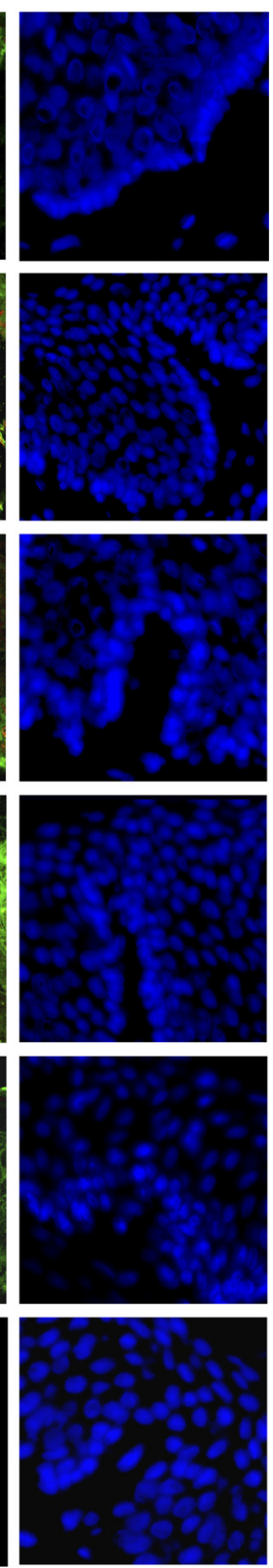

Figure 4. Microscopic colocalization of IFITM1, ITM2A, SPON1, DKK4, and CXCR4 with C/EBP $\delta$ in the limbus. First column: IFITM1, ITM2A, SPON1, DKK4, and CXCR4 signals (green). Second column: C/EBPS (red). Third column: overlay to show colocalization of $\mathrm{C} / \mathrm{EBP} \delta$ with the differentially expressed gene product. IFITM1-positive limbal basal cells are also positive for C/EBP $\delta$. Many, but not all, ITM2Aand SPON1-positive cells are also positive for $\mathrm{C} / \mathrm{EBP} \delta$. Cells that stain positively for DKK 4, CXCR4 include those limbal basal cells positive for C/EBP $\delta$ and many suprabasal cells that do not contain detectable amounts of $\mathrm{C} / \mathrm{EBP} \delta$. Fourth column: nuclear staining with DAPI (blue). Last row: isotype controls with DAPI staining, imaged with the highest gain settings in each column. Images are from $110 \times 110-\mu \mathrm{m}$ fields of view, except second row $(160 \times 160 \mu \mathrm{m})$. markers that could help limbal stem cell identification with flow cytometry, thus facilitating the production of stem cellenriched limbal populations.

Based on these criteria, we chose eight DEGs for further examination. Differential expression of all these molecules was confirmed by qRT-PCR. We used immunofluorescence microscopy to identify the protein product of five DEGs in the cornea and to explore their relation to putative resting stem cells. First we compared the limbal localization of some widely used and recently suggested stem cell markers. We showed the presence of a putative stem cell marker, Bmi-1, in the basal limbal cells; however, it was also present in superficial limbal epithelial cells, which is similar to its distribution in the epidermis, where its role in preventing premature differentiation of suprabasal epithelial cells was suggested in addition to stem cell maintenance. ${ }^{22}$ We showed that $\mathrm{C} / \mathrm{EBP} \delta$ colocalizes with membranous ABCG2 staining and a subpopulation of $\Delta$ Np63-containing basal limbal epithelial cells, which supports its previ- ously reported presence in mitotically quiescent limbal epithelial stem cells. ${ }^{18}$ We found $\Delta$ Np63 present in suprabasal cells, too, probably because $\Delta \mathrm{Np} 63$ is present in activated stem cells. ${ }^{16}$ In our experimental setting, C/EBP $\delta$ has proven to be highly useful for the detection of putative resting stem cells based on its reliable tissue staining pattern and exact colocalization with membranous ABCG2 staining. It must be noted that in other settings, especially when detecting proliferating stem cells in cultured epithelial sheets, p63 can be a more suitable marker because $\mathrm{C} / \mathrm{EBP} \delta$-positive resting stem cells are rare under tissue culture conditions. ${ }^{18,19,23}$

We showed that IFITM1, ITM2A, and SPON1 proteins localized primarily to C/EBP $\delta$-positive basal limbal epithelial cells. This finding suggests that these molecules can be stem cell-associated molecules, though each was present in cell types other than putative limbal epithelial stem cells. IFITM1 staining was seen in limbal stromal cells, which does not contradict its supposed stem cell association, because sev- 
eral putative stem cell types were described in the limbal stroma. ${ }^{24-26}$ However, further investigations are needed to elucidate the nature of IFITM1-positive limbal stromal cells. In addition to putative limbal stem cells, ITM2A and SPON1 localized to some suprabasal cells, indicating their involvement in early differentiation similar to that observed in epiphyseal cartilage differentiation for ITM2A. ${ }^{27}$ ITM2A was seen in the nuclei of some central corneal cells, which can be explained by the presence of a leucine zipper motif in the molecule. ${ }^{28}$

The association of these molecules with other adult stem cell types has been reported. IFITM family members were suggested to be targets of Wnt signaling in the intestinal stem cell compartment. ${ }^{29}$ ITM2A was shown in early differentiating chondroblasts and in neonatal mouse hair follicles. ${ }^{27}$ SPON1 promotes the differentiation of neural precursors ${ }^{30}$ and is able to support the survival of neuroblastoma cells under adverse conditions. $^{31}$ Its expression is induced by the transcription factor PAX6, ${ }^{32}$ which plays an essential role in the morphogenesis of limbal structures. ${ }^{33}$

Immunofluorescent microscopy showed that in spite of suggestive mRNA expression and data in the literature, the protein product of other DEGs was present in a considerably larger population of cells than the putative limbal epithelial stem cell compartment.

DKK4 was present in the suprabasal layers of the limbal epithelium, but it was not seen in central corneal cells. DKK4 is a member of a family of Wnt inhibitors comprising the DKK1-4 proteins. Dhouailly et al. ${ }^{34}$ suggested that inhibition of the Wnt pathway is required for the morphogenesis of corneal epithelium. This hypothesis is supported by the appearance in DKK2 knockout mice of skin-like corneal epithelium that actually forms hair follicles. ${ }^{35}$ Increased expression of DKK3 in human corneal versus conjunctival epithelium was also observed. ${ }^{36}$ The localization of DKK 4 in the limbus suggests that it may contribute to directing the differentiation of ocular surface epithelial cells toward the corneal phenotype.

CXCR4 staining in the basal and suprabasal limbal epithelium and basal central corneal cells indicates its role in differentiating transient amplifying cells (TACs). CXCR4 is the receptor of CXCL12/SDF1 (C-X-C chemokine ligand 12/stromal-derived factor 1) and participates in many cellular processes, including hematopoietic stem cell homing ${ }^{37}$ and epithelial cell proliferation. ${ }^{38}$ Recently, its differential expression was observed in porcine limbal side population cells. ${ }^{21}$ Based on these data, we suggest that CXCR4 may participate in maintaining the proliferative capacity of corneal limbal progenitors and TACs of the central corneal epithelium.

In summary, we have set up a database of differentially expressed genes of the human limbal epithelium. Our results contribute to the establishment of the molecular signature of limbal epithelial cells and provide tools for their more precise characterization. We have highlighted molecules (CXCR4, DKK4) whose role in the differentiation and maintenance of corneal epithelial cells can be assumed. Further investigations are needed to find out how they exert their functions and how their actions can be exploited in corneal epithelial cell culturing or healing corneal epithelial defects. We identified three molecules (SPON1, ITM2A, IFITM1) whose colocalization with $\mathrm{C} / \mathrm{EBP} \delta$-positive putative limbal epithelial stem cells indicates their potential usefulness in the histologic characterization of the limbal stem cell compartment and in situ tracking of the fates of putative stem and progenitor cells. Two of these molecules, ITM2A and IFITM1, are localized to the cell membrane and may be used as targets in sorting cells for expression profiling and cell biology studies.

\section{References}

1. Davanger M, Evensen A. Role of the pericorneal papillary structure in renewal of corneal epithelium. Nature. 1971;229:560-561.

2. Dua HS, Shanmuganathan VA, Powell-Richards AO, Tighe PJ, Joseph A. Limbal epithelial crypts: a novel anatomical structure and a putative limbal stem cell niche. Br J Opbthalmol. 2005;89: 529-532.

3. Shortt AJ, Secker GA, Munro PM, Khaw PT, Tuft SJ, Daniels JT. Characterization of the limbal epithelial stem cell niche: novel imaging techniques permit in vivo observation and targeted biopsy of limbal epithelial stem cells. Stem Cells. 2007;25:1402-1409.

4. Pellegrini G, Traverso CE, Franzi AT, Zingirian M, Cancedda R, De Luca M. Long-term restoration of damaged corneal surfaces with autologous cultivated corneal epithelium. Lancet. 1997;349:990 993.

5. Shortt AJ, Secker GA, Notara MD, et al. Transplantation of ex vivo cultured limbal epithelial stem cells: a review of techniques and clinical results. Surv Opbthalmol. 2007;52:483-502.

6. Takács L, Tóth E, Berta A, Vereb G. Stem cells of the adult cornea: from cytometric markers to therapeutic applications. Cytometry A. 2009;75:54-66.

7. Lavker RM, Sun TT. Epithelial stem cells: the eye provides a vision Eye. 2003;17:937-942.

8. Blanpain C, Lowry WE, Geoghegan A, Polak L, Fuchs E. Selfrenewal, multipotency, and the existence of two cell populations within an epithelial stem cell niche. Cell. 2004;118:635-648.

9. Tumbar T, Guasch G, Greco V, et al. Defining the epithelial stem cell niche in skin. Science. 2004;303:359-363.

10. Figueira EC, Di Girolamo N, Coroneo MT, Wakefield D. The phenotype of limbal epithelial stem cells. Invest Opbthalmol Vis Sci. 2007; 48:144-156.

11. Zhou M, Li XM, Lavker RM. Transcriptional profiling of enriched populations of stem cells versus transient amplifying cells: a comparison of limbal and corneal epithelial basal cells. J Biol Chem. 2006;281:19600-19609.

12. Budak MT, Alpdogan OS, Zhou M, Lavker RM, Akinci MA, Wolosin JM. Ocular surface epithelia contain ABCG2-dependent side population cells exhibiting features associated with stem cells. $J$ Cell Sci. 2005;118:1715-1724.

13. de Paiva CS, Chen Z, Corrales RM, Pflugfelder SC, Li DQ. ABCG2 transporter identifies a population of clonogenic human limbal epithelial cells. Stem Cells. 2005;23:63-73.

14. Schlotzer-Schrehardt U, Kruse FE. Identification and characterization of limbal stem cells. Exp Eye Res. 2005;81:247-264.

15. Wang DY, Cheng CC, Kao MH, Hsueh YJ, Ma DH, Chen JK. Regulation of limbal keratinocyte proliferation and differentiation by TAp63 and DeltaNp63 transcription factors. Invest Ophthalmol Vis Sci. 2005;46:3102-3108.

16. Di Iorio E, Barbaro V, Ruzza A, Ponzin D, Pellegrini G, De Luca M. Isoforms of DeltaNp63 and the migration of ocular limbal cells in human corneal regeneration. Proc Natl Acad Sci US A. 2005;102: 9523-9528.

17. Umemoto $\mathrm{T}$, Yamato $\mathrm{M}$, Nishida $\mathrm{K}$, Yang $\mathrm{J}$, Tano $\mathrm{Y}$, Okano $\mathrm{T}$. Limbal epithelial side-population cells have stem cell-like properties, including quiescent state. Stem Cells. 2006;24:86-94.

18. Barbaro V, Testa A, Di Iorio E, Mavilio F, Pellegrini G, De Luca M. C/EBPdelta regulates cell cycle and self-renewal of human limbal stem cells. J Cell Biol. 2007;177:1037-1049.

19. Rama P, Matuska S, Paganoni G, Spinelli A, De Luca M, Pellegrini G. Limbal stem-cell therapy and long-term corneal regeneration. N Engl J Med. 2010;363:147-155.

20. Ding Z, Dong J, Liu J, Deng SX. Preferential gene expression in the limbus of the vervet monkey. Mol Vis. 2008;14:2031-2041.

21. Akinci MA, Turner H, Taveras M, Wolosin JM. Differential gene expression in the pig limbal side population; implications for stem cell cycling, replication and survival. Invest Opbthalmol Vis Sci. 2009;50:5630-5638.

22. Lee $\mathrm{K}$, Adhikary G, Balasubramanian S, et al. Expression of Bmi-1 in epidermis enhances cell survival by altering cell cycle regulatory protein expression and inhibiting apoptosis. I Invest Dermatol. 2008;128:9-17. 
23. Salehi-Had H, Alvarenga LS, Isseroff R, Schwab IR. Factors modulating p63 expression in cultured limbal epithelial cells. Cornea. 2005;24:845-852.

24. Dravida S, Pal R, Khanna A, Tipnis SP, Ravindran G, Khan F. The transdifferentiation potential of limbal fibroblast-like cells. Brain Res Dev Brain Res. 2005;160:239-251.

25. Du Y, Funderburgh ML, Mann MM, SundarRaj N, Funderburgh JL. Multipotent stem cells in human corneal stroma. Stem Cells. 2005; 23:1266-1275.

26. Yoshida S, Shimmura S, Nagoshi N, et al. Isolation of multipotent neural crest-derived stem cells from the adult mouse cornea. Stem Cells. 2006;24:2714-2722.

27. Van den Plas D, Merregaert J. In vitro studies on Itm2a reveal its involvement in early stages of the chondrogenic differentiation pathway. Biol Cell. 2004;96:463-470.

28. Deleersnijder W, Hong G, Cortvrindt R, et al. Isolation of markers for chondro-osteogenic differentiation using cDNA library subtraction. J Biol Chem. 1996;271:19475-19482.

29. Andreu P, Colnot S, Godard C, et al. Identification of the IFITM family as a new molecular marker in human colorectal tumors. Cancer Res. 2006;66:1949-1955.

30. Schubert D, Klar A, Park M, Dargusch R, Fischer WH. F-spondin promotes nerve precursor differentiation. J Neurochem. 2006;96: $444-453$.
31. Cheng Y-C, Liang C-M, Chen Y-P, Tsai I-H, Kuo C-C, Liang S-M F-spondin plays a critical role in murine neuroblastoma survival by maintaining IL-6 expression. J Neurochem. 2009;110:947-955.

32. Wolf LV, Yang Y, Wang J, et al. Identification of Pax6-dependent gene regulatory networks in the mouse lens. PLOS ONE. 2009;4:e4159.

33. Lyngholm M, Hoyer PE, Vorum H, Nielsen K, Ehlers N, Mollgard K Immunohistochemical markers for corneal stem cells in the early developing human eye. Exp Eye Res. 2008;87:115-121.

34. Dhouailly D. A new scenario for the evolutionary origin of hair, feather, and avian scales. J Anat. 2009;214:587-606.

35. Mukhopadhyay M, Gorivodsky M, Shtrom S, et al. Dkk2 plays an essential role in the corneal fate of the ocular surface epithelium. Development. 2006;133:2149-2154.

36. Turner HC, Budak MT, Akinci MAM, Wolosin JM. Comparative analysis of human conjunctival and corneal epithelial gene expression with oligonucleotide microarrays. Invest Opbthalmol Vis Sci. 2007;48:2050-2061.

37. Zuba-Surma EK, Kucia M, Ratajczak J, Ratajczak MZ. "Small stem cells" in adult tissues: very small embryonic-like stem cells stand up! Cytometry A. 2009;75:4-13.

38. Florin L, Maas-Szabowski N, Werner S, Szabowski A, Angel P. Increased keratinocyte proliferation by JUN-dependent expression of PTN and SDF-1 in fibroblasts. J Cell Sci. 2005;118:19811989. 